Indonesian Journal of Physics

Vol 18 No. 2, April 2007

\title{
Corrosion Study of Steels In Liquid Lead-Bismuth Cooled Nuclear Reactors by Computer Simulation using Moldy Code
}

\author{
Alan Maulana ${ }^{1,4)}$, Zaki Su'ud ${ }^{1)}$, Hermawan K.D. ${ }^{3)}$, and Khairurrijal ${ }^{2)}$ \\ ${ }^{1)}$ Nuclear Physics and Biophysics Research Division \\ ${ }^{2)}$ Physics of Electronic Materials Research Division, \\ Faculty of Mathematics and Natural Sciences, \\ ${ }^{3)}$ Engineering Physics Research Division, \\ Faculty of Industrial Technology \\ Institut Teknologi Bandung, Indonesia \\ ${ }^{4)}$ Neutron Scattering Laboratory PTBIN-BATAN \\ Kawasan Puspiptek Serpong Cisauk-Tangerang 15314, Indonesia \\ email :alan.maulana@students.itb.ac.id
}

\begin{abstract}
The corrosion of steel in liquid lead-bismuth has been studied by computer simulation using MOLDY code. The diffusion processes among atoms are assumed to be the key issues to understand the corrosion mechanism microscopically. In order to simulate these diffusion processes, the inter-atomic potential between $\mathrm{Fe}-\mathrm{Fe}, \mathrm{Pb}-\mathrm{Pb}$, $\mathrm{Bi}-\mathrm{Bi}, \mathrm{Cr}-\mathrm{Cr}$ and $\mathrm{Ni}-\mathrm{Ni}$ are assumed to obey the Lennard-Jones potential. The Lennard-Jones potential parameters of above pairs of atom have been derived by fitting the data available in the literature with the Lennard-Jones equation. The initial positions of the system are taken from the crystal structures data including the cell parameters. The simulation cell was a box with the volume 60x30x30 $\AA^{3}$ that were filled by 2864 atoms. Nickel and chromium atoms were substituted into Fe crystal with the percentage $10 \%$ and $16 \%$ respectively to construct the systems of SS-316 which contacted by 50\% Pb-50\%Bi. The molecular dynamic simulations have been carried out for surface interaction between steel crystal with liquid lead-bismuth for several temperatures. After done the above simulation, the simulation is then tried by using $45 \% \mathrm{~Pb}-55 \% \mathrm{Bi}(\mathrm{Pb}-\mathrm{Bi}$ Eutectic). The results of molecular dynamic simulations for several temperatures and the effect of variation of $\mathrm{Pb}$ and $\mathrm{Bi}$ above will be presented in this paper.
\end{abstract}

Keywords: molecular dynamic, Lennard-Jones potential, liquid lead-bismuth, stainless steel, corrosion, fast breeder reactor, penetration depth.

\section{Introduction}

The liquid lead-bismuth eutectic (LBE) is one of the candidate coolants in Generation IV nuclear reactor especially for fast reactor or fast breeder reactor (FBR). The application of this material have many advantages because of low melting point, very high boiling temperatures and chemical inertness that can contributes to the safety inherent with liquid metal reactor (LMR). However, there is a problem of low corrosion resistance of the materials structure in contact with this liquid metal. Although some of the corrosion problem has been overcome by film coating in the surface of steels and flowing oxygen controlled in the system, there are some efforts to understand the corrosion phenomena macroscopically or microscopically.

Many investigations of corrosion phenomena of stainless steel (SS) in liquid $\mathrm{Pb}-\mathrm{Bi}$ has been carried out macroscopically by experiment, nevertheless there were some studies done by using computer simulation. Among those studies contributions by the groups from Tokyo Institute of Technology (Tokyotech/TIT) and University of Nevada are important $^{1-3)}$.

The group from University of Nevada in 2002 had investigated corrosion phenomena using computational chemical kinetics and hydrodynamics methods. Meanwhile, the group from Tokyotech carried out the studies using molecular dynamics methods. Their interatomic potential for $\mathrm{Fe}-\mathrm{Fe}, \mathrm{Pb}-\mathrm{Pb}$, $\mathrm{Bi}-\mathrm{Bi}, \mathrm{Fe}-\mathrm{Pb}, \mathrm{Fe}-\mathrm{Bi}$ and $\mathrm{Pb}-\mathrm{Bi}$ were derived by using ab-initio method. The depth of potential well was used to analyze the cohesive forces between atoms. The deeper the potential depth, the higher the cohesive forces and therefore the more difficult diffusion process to occur.

The group from Tokyotech also had published the paper about interaction between $\mathrm{Pb}$ atom and/or $\mathrm{Bi}$ atom which located at the surface of the Fe (iron) crystal at high temperatures using first principle molecular dynamic. The result of this simulation showed the motion of $\mathrm{Pb}$ atom and $\mathrm{Bi}$ atom on $\mathrm{Fe}$ crystal surface.

However, since they used ab-initio method that computationally very expensive then only a few atoms were involved in their simulation. Therefore in order to accommodate more atoms, we used simple interatomic potential to be used with molecular dynamic methods at reasonable computational time. We used Lennard-Jones potential in this simulation. Some of $\mathrm{Fe}$ atoms in $\mathrm{Fe}$ crystal were substituted by nickel and chromium atoms to represent SS 316. 
Basically a corrosion phenomenon in this regard is indicated by the penetration of $\mathrm{Pb} / \mathrm{Bi}$ into stainless steel. Our simulation results showed the existence of this penetration process.

Molecular dynamics simulation is a technique for computing the equilibrium and transport properties of classical many-body system. This is an excellent approximation for a wide range materials simulations. Basically Molecular dynamics is a method to calculate positions and velocities of molecules or atoms as a function of time-step. All positions and velocities data calculated are called phase space trajectories which can be used to determine the macroscopic properties of materials. This method using finite different method to solve Newton's second law of motion ${ }^{4,5)}$.

In this study, we try to study corrosion phenomena of SS 316 in contact with $\mathrm{Pb}-\mathrm{Bi}$ at several temperatures. The simulations use MOLDY code, an open source molecular dynamics software, which can be used to simulate solid or liquid system. The computer experiment were done by varying the percentage of $\mathrm{Pb}$ and $\mathrm{Bi}$ composition in the $\mathrm{Pb}-\mathrm{Bi}$ system in contact with SS 316. The percentage of $\mathrm{Pb}$ $\mathrm{Bi}$ are $50 \% \mathrm{~Pb}-50 \% \mathrm{Bi}$ and $45 \% \mathrm{~Pb}-55 \% \mathrm{Bi}(\mathrm{Pb}-\mathrm{Bi}$ Eutectic).

\section{Theory}

Basically molecular dynamic simulation divides into two tasks: developing a model and using a model in a simulation. The first task includes choosing a form for the intermolecular potential and then deriving appropriate equations of motion. The second task divides into two parts: generating phasespace trajectories and analyzing the trajectories for properties.

In the first step in performing a simulation, we must choose a functional form for the intermolecular potential. As mentioned above that we used LennardJones equation for the intermolecular potential between pair of atoms:

$$
u(r)=4 \varepsilon\left[\left(\frac{\sigma}{r}\right)^{12}-\left(\frac{\sigma}{r}\right)^{6}\right]
$$

where $r$ is the atomics distance, $\varepsilon$ the potential depth and $\sigma$ potential parameter.

To calculate the parameters $\varepsilon$ and $\sigma$ for different types of atoms we used the Lorentz-Berthelet mixing rule equation as follows ${ }^{6}$ :

$$
\begin{aligned}
& \sigma_{A B}=0.5\left(\sigma_{A A}+\sigma_{B B}\right), \\
& \varepsilon_{A B}=\left(\varepsilon_{A A} \varepsilon_{B B}\right)^{1 / 2} .
\end{aligned}
$$

The forces based on the Lennard-Jones potential above are :

$$
\vec{F}=-\frac{d u(r)}{d r}=24 \frac{\varepsilon}{\sigma}\left[2\left(\frac{\sigma}{r}\right)^{13}-\left(\frac{\sigma}{r}\right)^{7}\right]
$$

and

$$
\vec{F}_{i}=\sum_{j \neq i}^{N} \vec{F}_{i j} ;(i, j=1, \ldots, N),
$$

where $\vec{F}_{i}$ is the sum of forces $\vec{F}_{i j}$ on atom $i$ due to atom $j(j \neq i)$ in the system. $N$ is the number atoms involved in the simulation. These atoms are assumed to be classical and therefore obey the classical mechanics. The equation of motion can be written as follow :

$$
\vec{F}_{i}=m_{i} \frac{\partial \vec{v}_{i}}{\partial t}=m_{i} \frac{\partial \vec{r}_{i}}{\partial t} \quad ;(i=1, \ldots, N),
$$

where $m_{i}$ is atomic mass of particle $i, v_{i}$ and $r_{i}$ respectively velocity and position of atom $i$. Equation (6) can be solved numerically by finite different method. Some methods to solve this equation among others are the well known leap-frog algorithm, Verlet algorithm, Gear predictor-corrector, Beeman etc. In this study we used Beeman algorithm.

The energy or forces calculation is the most time-consuming part almost all Molecular Dynamics simulations. If we consider a model system with pairwise interactions, we have to consider the contribution to the force on particle $i$, by all neighbors. For a system of $N$ particles, we must evaluate $\mathrm{N}(\mathrm{N}-1) / 2$ pair interactions ${ }^{5)}$. This implies that the time needed for evaluation of the energy scale as $N^{2}$. This process is too long in the simulation. There exist efficient techniques for speeding up the evaluation. Some of the techniques are called Verlet list and Cell (or linked list).

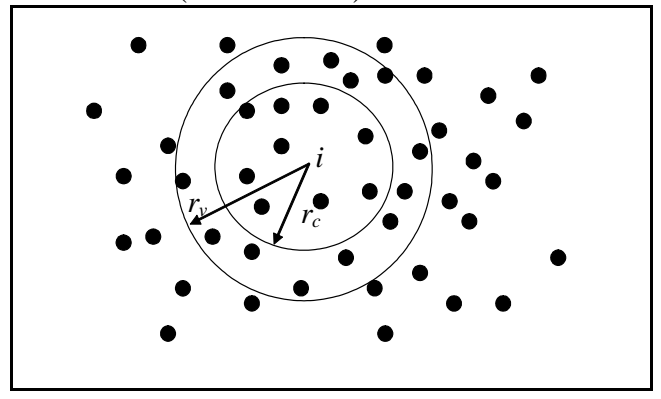

Figure 1. The Verlet list method

Verlet develop a bookkeeping technique which is illustrated in Figure 1 above $e^{4,5)}$. In this method a second radius cutoff $r_{\mathrm{v}}>r_{\mathrm{c}}$ is introduced, where $r_{\mathrm{c}}$ is first radius cutoff. Before the interactions was calculated, firstly a list of all particles within a radius $r_{\mathrm{v}}$ of particle $i$ was made (Verlet list). In the subsequent calculation of the interactions, only those particles in this list have to be considered. This is a calculation of order $\mathrm{N}$. As soon as one of the particles is displaced more than $r_{\mathrm{v}}>r_{\mathrm{c}}$, a list have to update.

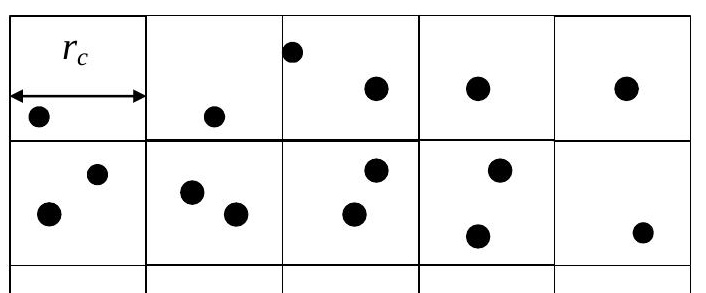


Figure 2. The cell list method

The idea of cell list is illustrated in Figure 2. The simulation box is divided into cells with a size equal or slightly larger than the cutoff radius $r_{\mathrm{c}}$. Each particles in a given cell interact with only those particles in the same or neighboring cells. Since the allocation of a particle to a cell is an operation that scales with $N$ and the total number of cells that needs to be considered for the calculation of the interaction is independent of the system size, the cell list method scales as $N$. Moldy code used cell list to gain the speed of calculation forces or energy.

\section{Methodology}

The simulation requires the initial condition of positions and velocities of all atoms involved in the system. The initial positions of Fe was developed based on the BCC structure with lattice parameter $2.8665 \AA$ and $\mathrm{Pb}$ atoms are determined to fill the FCC cell with lattice parameter $4.9508 \AA$ as illustrated in Figure 3.

The unit cell of the systems can be extended periodically in three dimensions with the equation:

$$
\vec{r}=\vec{r}_{u c}+n_{x} \vec{a}+n_{y} \vec{b}+n_{z} \vec{c},
$$

where $\vec{r}_{u c}$ is the vector position of unit cell, $n_{x}, n_{y}, n_{z}$ are the number of cell in the $\mathrm{x}, \mathrm{y}, \mathrm{z}$ directions, $\vec{a}, \vec{b}$ and $\vec{C}$ are the unit vector in the Cartesian coordinates. Nickels and chromiums were randomly added to the pure Fe structure above as impurities to develope Fe alloy structure. Some of bismuth atoms also replaced of some $\mathrm{Pb}$ atoms in the pure $\mathrm{Pb}$ structure. The initial velocities of each atom were generated randomly with the value between 1 to -1 .

The parameters Lennard-Jones used in our simulation are taken from the data of energy potential versus interatomic distance of the same atoms in the literature ${ }^{1,7,8)}$. These data are fitted by the LennardJones equation. The results are shown in Table 1.
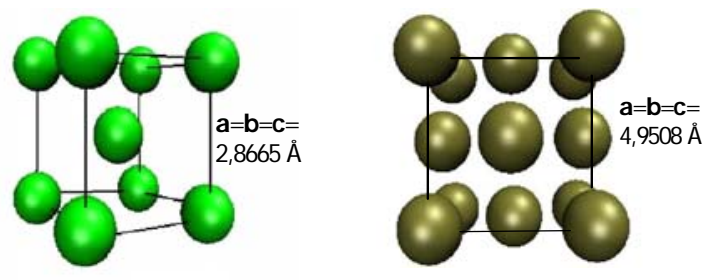

Figure 3. Lattice constant of (a) Fe, (b) Pb, (c) Bi and $\mathrm{Pb}-\mathrm{Bi}$

Table 1. Lennard-Jones parameter used in the simulation

\begin{tabular}{|c|c|c|c|}
\hline No & Element & $\varepsilon(\mathrm{eV})$ & $\sigma(\AA)$ \\
\hline 1 & $\mathrm{Fe}-\mathrm{Fe}$ & -0.62 & 2.26 \\
\hline 2 & $\mathrm{~Pb}-\mathrm{Pb}$ & -0.125 & 2.75 \\
\hline 3 & $\mathrm{Bi}-\mathrm{Bi}$ & -0.075 & 2.8 \\
\hline \hline 4 & $\mathrm{Ni}-\mathrm{Ni}$ & -0.6 & 1.9 \\
\hline 5 & $\mathrm{Cr}-\mathrm{Cr}$ & -0.2 & 2.5 \\
\hline
\end{tabular}

\section{Results and Discussion}

Figures 4 shows the atomics positions of $\mathrm{Fe}-$ $10 \% \mathrm{Ni}-16 \% \mathrm{Cr}$ in contact with $50 \% \mathrm{~Pb}-50 \% \mathrm{Bi}$ after molecular dynamics simulations in 20 thousand timesteps at several temperatures. These figure presents the atomic positions of Fe (Green), nickel (blue), chromium (grey), $\mathrm{Pb}$ (Brown) and $\mathrm{Bi}$ (white) after molecular dynamics simulation at several temperatures. It can be shown that some of $\mathrm{Pb}$ and $\mathrm{Bi}$ atoms can diffuse into Fe system. The penetration depth of $\mathrm{Pb}$ and $\mathrm{Bi}$ are shown in Figure 5, 6 and 7.

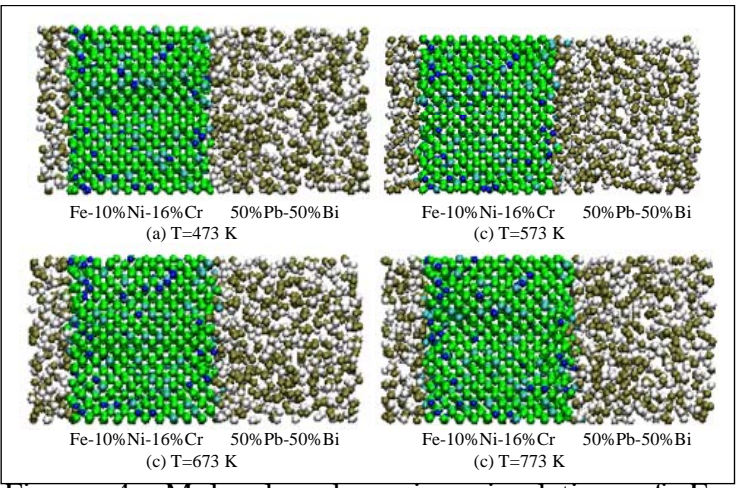

Figure 4. Molecular dynamics simulation of $\mathrm{Fe}$ systems with $50 \% \mathrm{~Pb}-50 \% \mathrm{Bi}$ at several temperatures. 


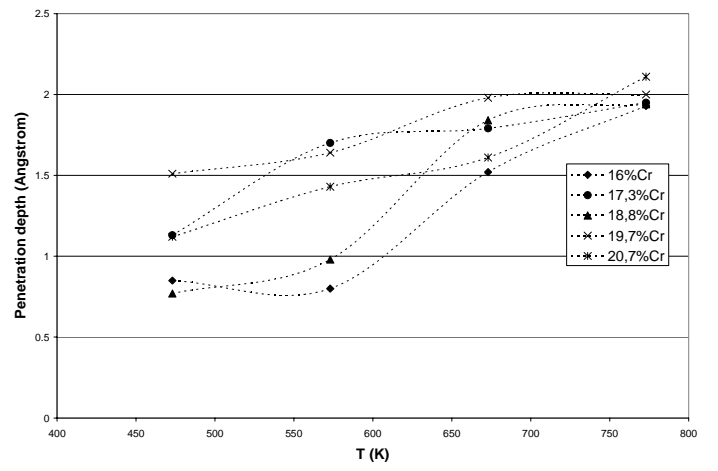

Figure 5. Penetration depth of $\mathrm{Pb}$ as a function of temperatures in Fe system with different composition of chromium.

Figures 5 and 6 shows that generally the penetration depth of $\mathrm{Pb}$ and $\mathrm{Bi}$ increase with increasing the temperatures. Figure 5 also indicate that the composition of chromium $16 \%$ of Fe system show the lowest penetration depth of $\mathrm{Pb}$.

The penetration depth of $\mathrm{Pb}$ and $\mathrm{Bi}$ as a function of the composition of chromium at $773 \mathrm{~K}$ are presented in Figure 7. At this temperature the penetration depth of $\mathrm{Pb}$ and $\mathrm{Bi}$ increase with increasing the composition of chromium. The penetration depth of $\mathrm{Pb}$ is also more higher than $\mathrm{Bi}$.

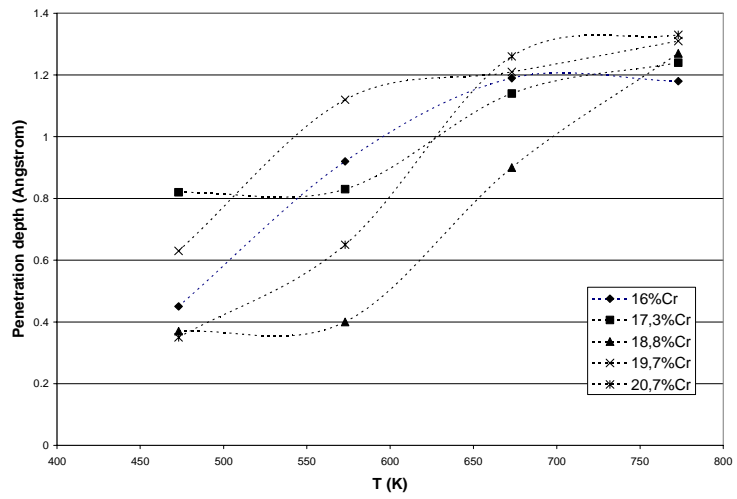

Figure 6. Penetration depth of $\mathrm{Bi}$ as a function of temperatures in Fe system with different composition of chromium.

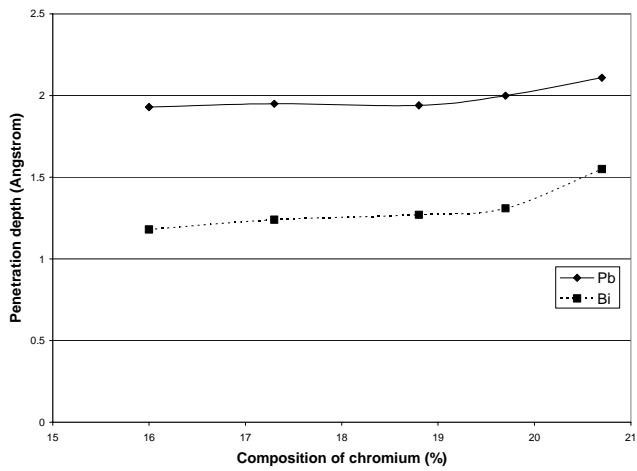

Figure 7. Penetration depth of $\mathrm{Pb}$ and $\mathrm{Bi}$ as a function of composition of chromium at $773 \mathrm{~K}$.

The results of molecular dynamic simulations of contact between $\mathrm{Fe}$ system and $\mathrm{Pb}-\mathrm{Bi}$ eutectic $(45 \% \mathrm{~Pb}-55 \% \mathrm{Bi})$ at $773 \mathrm{~K}$ are illustrated in Figure 8 and 9. In this simulation the composition of chromium in the $\mathrm{Fe}-10 \% \mathrm{Ni}$ system are varied from $13 \%$ to $20.3 \%$. It can be shown that the surface of the Fe$10 \% \mathrm{Ni}$ systems can be penetrated by the leads and bismuths atoms. The penetrations depth of $\mathrm{Pb}$ and $\mathrm{Bi}$ into $\mathrm{Fe}-10 \% \mathrm{Ni}$ system is presented in Figure 10.

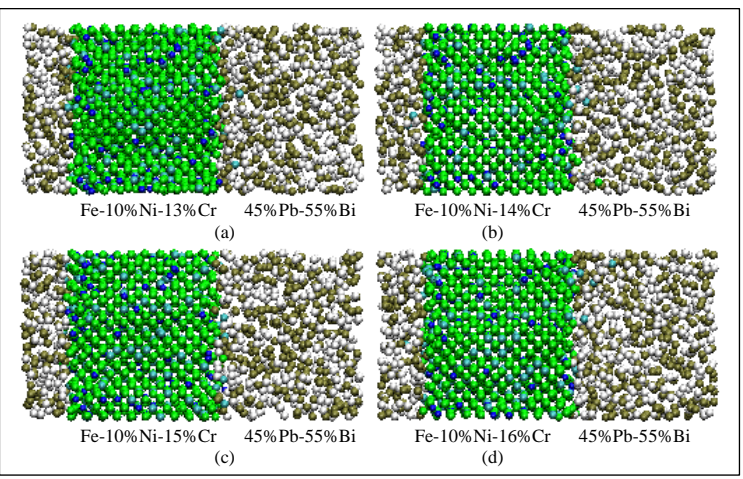

Figure 8 . The atomics posistion of $45 \% \mathrm{~Pb}-55 \% \mathrm{Bi}$ and Fe-10\%Ni systems after molecular dynamic simulation at $773 \mathrm{~K}$

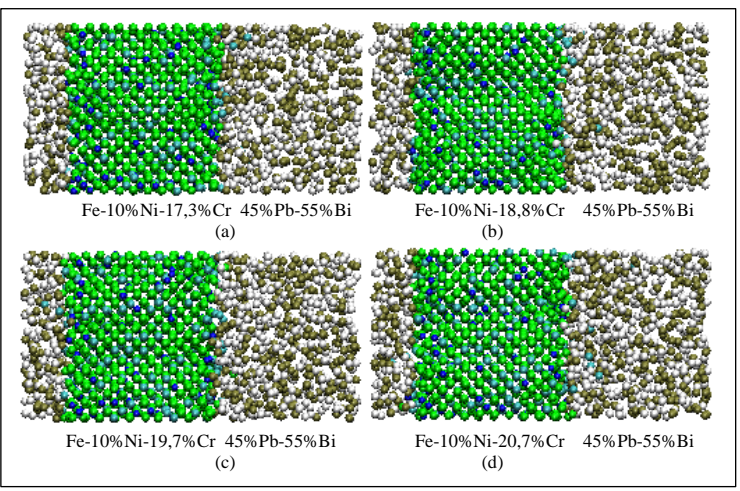

Figure 10 . The atomics posistion of $45 \% \mathrm{~Pb}-55 \% \mathrm{Bi}$ and $\mathrm{Fe}-10 \% \mathrm{Ni}$ systems after molecular dynamic simulation at $773 \mathrm{~K}$. 


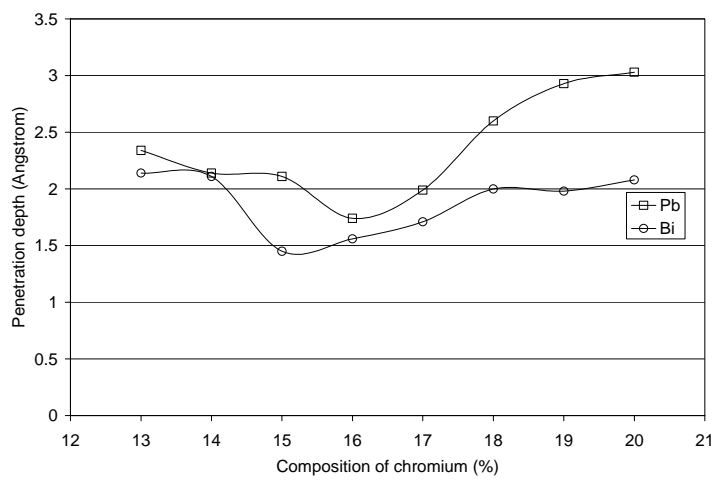

Figure 11. Penetration depth of $\mathrm{Pb}$ and $\mathrm{Bi}$ into Fe$10 \% \mathrm{Ni}$ with different contain of chromium at $773 \mathrm{~K}$.

Figure 10 shows that the composition of chromium $20.7 \%$ in the Fe system, the lead atoms can penetrate more deeper than the other about $3 \AA$. Penetration depth bismuth atoms generally more lower than leads atoms in all composition of chromium. The penetration depth of lead at $16 \%$ of chromium composition is more lower than the other.

The penetration depth of $\mathrm{Pb}$ and $\mathrm{Bi}$ in the interaction between $45 \% \mathrm{~Pb}-55 \% \mathrm{Bi}$ with $\mathrm{Fe}-10 \% \mathrm{Ni}-$ $16 \% \mathrm{Cr}$ as a function of temperatures are illustrated in Figure 11. The penetration depth of $\mathrm{Pb}$ and $\mathrm{Bi}$ atoms increases with increasing the temperatures.

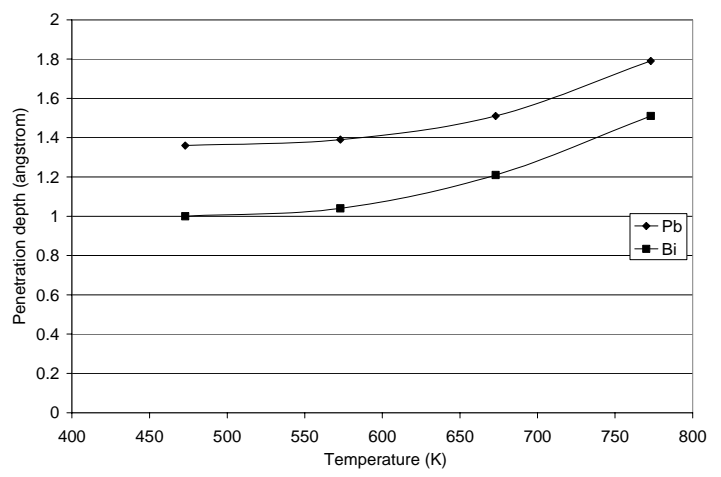

Figure 12. Penetration depth of $\mathrm{Pb}$ and $\mathrm{Bi}$ into Fe$10 \% \mathrm{Ni}-16 \% \mathrm{Cr}$ as a function of temperatures.

\section{Conclusions}

Molecular dynamic simulation can be use to study corrosion phenomena of stainless steel in liquid lead-bismuth through penetration of lead and bismuth atoms into Fe system.

The penetration depth of $\mathrm{Pb}$ and $\mathrm{Bi}$ increase with increasing temperatures. The penetration depth of $\mathrm{Pb}$ is more higher than $\mathrm{Bi}$ in the simulation of contact between $50 \% \mathrm{~Pb}-50 \% \mathrm{Bi}$ or $45 \% \mathrm{~Pb}-55 \% \mathrm{Bi}$ with Fe system.

The penetration depth of $\mathrm{Pb}$ and $\mathrm{Bi}$ in $\mathrm{Fe}$ system is depending on the composition of chromium.

\section{Acknowledgements}

These research supported by Ministry of Science and Technology of Republik Indonesia under Rintisan Gelar programme.

\section{References}

1. Y. Qi, and M. Takahashi, Study on Corrosion Phenomena of Steels in $\mathrm{Pb}-\mathrm{Bi}$ Flows, Proc. ICONE 11, 36375, Tokyo, Japan, 2003.

2. K. Refson, Moldy, a Portable Molecular Dynamics Simulation Program for Serial and Parallel Computers, Comput. Phys. Commun., 126(3), 309-328, 2000.

3. C. Wu et al., Numerical Modelling of Lead Oxidation in Controlled Lead Bismuth Eutectic Systems : Chemical Kinetics and Hydrodinamic Effect, Internatonal on Advanced Nuclear Power Plants in Hollywood, Florida, June 9-13, 2002.

4. D. Frenkel, and B. Smith, Understandings Molecular Simulation, Academic Press, 2002.

5. J. M. Haile, Molecular Dynamics Simulations, Elementary Methods, John Wiley \& Sons, NewYork, 1997.

6. E. R. Zhdanov, and Fakhretdinov, Molecular Dynamics Simulation of The Liquid-Vapor Interface of Binary mixtures, J. Mol. Liq., 120:13,.51-53, 2005.

7. A. Landa et al., Development of Finnish-Sinclair type potentials for $\mathrm{Pb}, \mathrm{Pb}-\mathrm{Bi}$ and $\mathrm{Pb}-\mathrm{Ni}$ systems : Application to Surface Segregation, Acta Mater., 46:9, 3027-3032, 1998.

8. P. Olsson et al., Two-Band Modeling of $\alpha$-Prime Phase Formation in Fe-Cr, Phys. Rev. B., 72, 214119, 2005.

9. D. Sapundjiev, S. Van Dyck and W. Bogaerts, Liquid Metal Corrosion of T91 and A316L materials in $\mathrm{Pb}-\mathrm{Bi}$ Eutectic at Temperatures 400 $600{ }^{\circ}$ C, Corros. Sci., 48, 577-594, 2006.

10. F. Balbaud-Celerier and F. Barbier, Investigation of Models to Predict the Corrosion of Steels in Flowing Liquid Lead Alloys, J. Nucl. Mater., 289, 227-242, 2001.

11. M. Cellino, R. Conversano and V. Rosato, Atomistic Simulation of Liquid Lead and Liquid Lead-bismuth Eutectic, J. Nucl. Mater., 301, 6469, 2002.

12. I. Kaban et al., Atomic Structure and Physical Properties of Liquid Pb-Bi Alloys, J PhysCondens Mat., 16, 6335-6341, 2004.

13. K. Hata, K. Hara, and M. Takahashi, Experimental Studies on Steels Corrosion in PbBi with Steam Injection, Progress in Nuclear Energy; 47:1-4,. 596-603, 2005.

14. K. Morita et al., Thermophysical Properties of Lead-Bismuth Eutectic Alloy For Use In Reactor Safety Analysis, Second Meeting of the NEA Nuclear Science Committee, France, September 23-24, 2004. 
15. M. Takahashi et al., First-Principle Molecular Dynamics Simulation on Interatomic Interaction of Fe Crystal with $\mathrm{Pb}$ and $\mathrm{Bi}$ atoms, Science and Technology of Advanced Materials, 5, 673-676, 2004. 